\title{
Time (also) flies from left to right
}

\author{
Julio Santiago, Juan Lupiáñez, Elvira Pérez and María Jesús Funes \\ University of Granada, Granada, Spain
}

\begin{abstract}
Everyday linguistic expressions in many languages suggest that back and front space is projected onto temporal concepts of past and future (as in the sentence we are years ahead of them). The present experiment tested the psychological reality of a different space-time conceptual metaphor-projecting the past to left space and the future to right space - for which there are no linguistic traces in any language. Participants categorized words as referring to the past or to the future. Irrelevant to this task, words appeared either to the left or right of the screen, and responses were given by keypresses of the left or right hand. Judgments were facilitated when word position and response mapping were congruent with the left-past right-future conceptual metaphor. These results are discussed in the context of current claims about the embodiment of meaning and the possible mechanisms by which conceptual metaphors can be acquired.
\end{abstract}

Everyday conversations provide many examples that seem to imply that people locate the past behind them and the future in front of them, and that they think of time as a movement from one (past) location to another (future) location (e.g., Back in the 60's, In the weeks ahead of us; Clark, 1973; Lakoff \& Johnson, 1980). Although there are alternative and incompatible ways of conceptualizing time (such as TIME IS MONEY, Lakoff \& Johnson, 1980; Levine, 1997), the spatial metaphor TIME IS MOVEMENT ALONG A PATH seems to be prevalent in many languages. Most of these languages use the horizontal back-front axis to map onto temporal "movement" and conceptualize the experiencer as moving forward from the past into the future: an "ego-moving" perspective (see Malotki, 1983, for Hopi; Alverson, 1994, for Mandarin, Hindi and Sesotho; Özçaliskan, 2003, for Turkish; see Radden, 2004, for a review of data from Western, Chinese, West African, and Indian languages). Alternatively, the experiencer may be seen as static, with future events frontally approaching and passing him or her by toward the back (a "time-moving" perspective; Clark, 1973). Núñez and Sweetser (2006) proposed a more elaborate taxonomy, emphasizing the importance of the reference point. In their classification, ego-moving and time-moving perspectives are subtypes of the ego-reference-point metaphors, in which the deictic origin is placed on the here and now of the speaker. There are also time-reference-point metaphors, in which the deictic origin is not part of the sequence of events, as in Christmas comes after Thanksgiving.

Within the conceptual metaphor view (Lakoff \& Johnson, 1980, 1999), researchers have suggested that abstract concepts (such as time) import their structure through conceptual projection from more concrete domains (such as space). The building blocks of all concepts are basic image schemas that arise from universal perceptuomotor experiences (Johnson, 1987; Mandler, 1992). The image schema acquired from forward movement of the body from one point to another probably constitutes the experiential basis for our spatialization of time along the back-front axis.

There are languages that resort to different ways to project space onto time, but again, the back-front axis is the most productive, since it affords the application of a greater number of image schemas. For example, in Toba (Klein, 1987) and Aymara (Núñez \& Sweetser, 2006), the past is in front of speakers because it can be "seen," whereas the future is behind because one cannot know ("see") clearly what will happen. The up-down axis is used to a limited extent in Western languages (as in the weeks coming up; Radden, 2004) and extensively in Chinese (Boroditsky, 2001; $\mathrm{Yu}, 1998)$. In contrast to the back-front and up-down axes, in his cross-linguistic review of spatial metaphors of time, Radden (2004) found no evidence whatsoever for any use of the left-right axis in lexical items, syntactic constructions, or any other kind of conventional linguistic marker of time: "[The left-right axis] does not seem to offer any sensible spatial basis for our understanding of time at all" (p. 227). Although languages often talk of the "approaching month," no language uses expressions like "the rightward month." Apparently, the only exception to this claim are signed languages, which conventionally refer to the left-right axis to place sequences of events (Emmorey, 2001).

In the psycholinguistic literature, there is already direct evidence available for the back-front time metaphor, both in its ego-moving and time-moving perspectives (Boroditsky, 2000; Boroditsky \& Ramscar, 2002; McGlone \& Harding, 1998), but there are virtually no studies testing the left-right mapping of time onto space. There are, however, some hints that such metaphors should be actually represented in semantic memory, at least in the minds of literate people from left-to-right writing cultures. In these cultures, not only text runs from left to right. There is also a highly conventional association of time to the horizontal axis in graphs, increasing from left to right; comic strips also flow from left to

J. Santiago, santiago@ugr.es 
right, and, in an interesting parallel of the contrast between the ego-moving and time-moving perspectives, the pages of books move from right to left as we advance in the story. Tversky, Kugelmass, and Winter (1991) asked American, Arab, and Hebrew children to place three stickers on a piece of paper to represent spatial (e.g., three peg dolls arranged in front of them), temporal (e.g., breakfast, lunch, and dinner times), preference (e.g., favorite food, a somewhat-liked food, and totally disliked food), and quantity concepts (e.g., a spoonful, a bucketful, and a whole dump truck full of sand). Only temporal concepts showed a clear linearity effect, with American children ordering temporal concepts from left to right and Arab children using a right to left order.

Placing stickers on paper is a highly off-line and reflexive task, but so far it is the only published experimental study on this issue. Less direct evidence is provided by the work of Chatterjee and others (Chatterjee, Maher, Gonzalez-Rothi \& Heilman, 1995; Chatterjee, Southwood, \& Basilico, 1999; Maher, Chatterjee, Gonzalez-Rothi, \& Heilman, 1995) who studied the flow of events, intimately connected to the flow of time. These authors showed that English speakers have a left-to-right bias when thinking of the agent and patient roles of a sentence: The agent tends to be imagined to the left of the patient. Moreover, actions like "push" or "pull" are also imagined flowing from left to right. By comparing native speakers of Arabic and Italian, Maas and Russo (2003) showed that this left-to-right bias is associated to writing direction.

Another line of indirect evidence is provided by number. It is well established that numerical sequences are represented along a spatial mental line running from left to right (Dehaene, Bossini, \& Giraux, 1993). People are faster to respond to small numbers with the left hand than the right hand, and to large numbers with the right hand than the left hand (the space number association of response codes [SNARC] effect). A similar effect has been shown with ordinal sequences, such as months of the year, letters in the alphabet (Gevers, Reynvoet, \& Fias, 2003), and days of the week (Gevers, Reynvoet, \& Fias, 2004). The directionality of the number line seems to be linked to writing direction, since it was also found that the effect faded and even reversed with users of right-to-left languages (Dehaene et al., 1993; Zebian, 2005).

Finally, Núñez and Sweetser (2006) gave an example of a speaker of Aymara gesturing from left to right simultaneously with an Aymara sentence literally meaning day goes, and day after day. The authors suggested that this type of left-to-right gesturing is common in speakers of many languages when time-reference-point metaphors are used. However, they did not provide statistical analyses of the frequency of occurrence of these gestures in the context of relevant expressions, and their methods were flawed in many other ways (e.g., they did not blind judges to the hypotheses of the study), factors that make this evidence remain at the anecdotal level.

In sum, there is suggestive evidence that literate users of left-to-right written languages map past time onto left space and future time onto right space. However, the present study is the first direct test of the automatic activation of the spatial left-right axis in the processing of temporal concepts. Our aim was to test the psychological reality of spatial con- ceptual metaphors of time and to do it precisely by looking for the metaphor that maps the past onto left space and the future onto right space, and of which there are no known linguistic traces. We used a simple and straightforward test of this idea. People were asked to judge whether words referred to the past or future. Completely irrelevant to this task, two left-right spatial dimensions were manipulated: Words could be presented to the left or right of the fixation point, and past and future responses could be mapped to the left and right hand, respectively, or have the contrary mapping. We chose to manipulate both spatial dimensions in order to increase our chances of finding the predicted interaction: Past words should be judged faster when presented to the left and/or responded to with the left hand, and future words should be judged faster when presented to the right and/or responded to with the right hand. If spatial dimensions affect categorization behavior in this situation, they are likely to do so by means of the automatic activation of spatial codes when thinking about past and future. At this point of our research, we will not discuss whether the relevant left-to-right metaphor uses ego as its reference point or not, since both possibilities will lead to the same prediction.

\section{METHOD}

\section{Participants}

Thirty-two University of Granada undergraduates participated in exchange for course credit. All of them were native Spanish speakers with normal or corrected-to-normal vision.

\section{Materials}

A total of 48 Spanish words were used, half of them referring to past time and the other half referring to future time (see the Appendix). Seventeen out of the 24 words per condition were tensed verbs (e.g., dijo, "he said"). Verb lemmas were the same across both the past and future conditions. They varied in person and number as well as in tense. Care was exercised to ensure that past and future tense could not be discerned by means of any other characteristic of the verb: Person and number varied randomly, and orthographic marks, such as the accent sign, occurred equally often on verb inflections across categories. The remaining words were temporal adverbs (e.g., mañana, "tomorrow") and the words pasado ("past") and futuro ("future"). Past and future words were matched in word frequency, length in letters and syllables, and stress placement.

\section{Apparatus}

The experiment was written in MicroExperimental (MEL) code (Schneider, 1988) and run on an IBM PS/2 30286 personal computer. Latencies were measured with millisecond precision.

\section{Procedure}

Participants were tested individually in a sound-insulated room. The instructions explained that words would be presented one by one on the screen and that their task was to decide whether the word referred to the past or the future. The instructions explicitly mentioned that the words would appear in different positions on the screen, but that this was irrelevant for the task. There were two response keys, " $\mathrm{f}$ " for the left hand and " $\mathrm{j}$ " for the right hand. The experiment consisted of two halves, which were perfect replications except for the response key to temporal meaning mapping (past to left key and future to right key was the congruent mapping, whereas the converse was the incongruent mapping). The order of presentation of key mapping was counterbalanced over participants. Each half consisted of instructions, 8 practice trials, and two blocks of 48 trials, which were presented with no break. Materials were divided into two lists that contained an equal number of past and future words. In the first ex- 

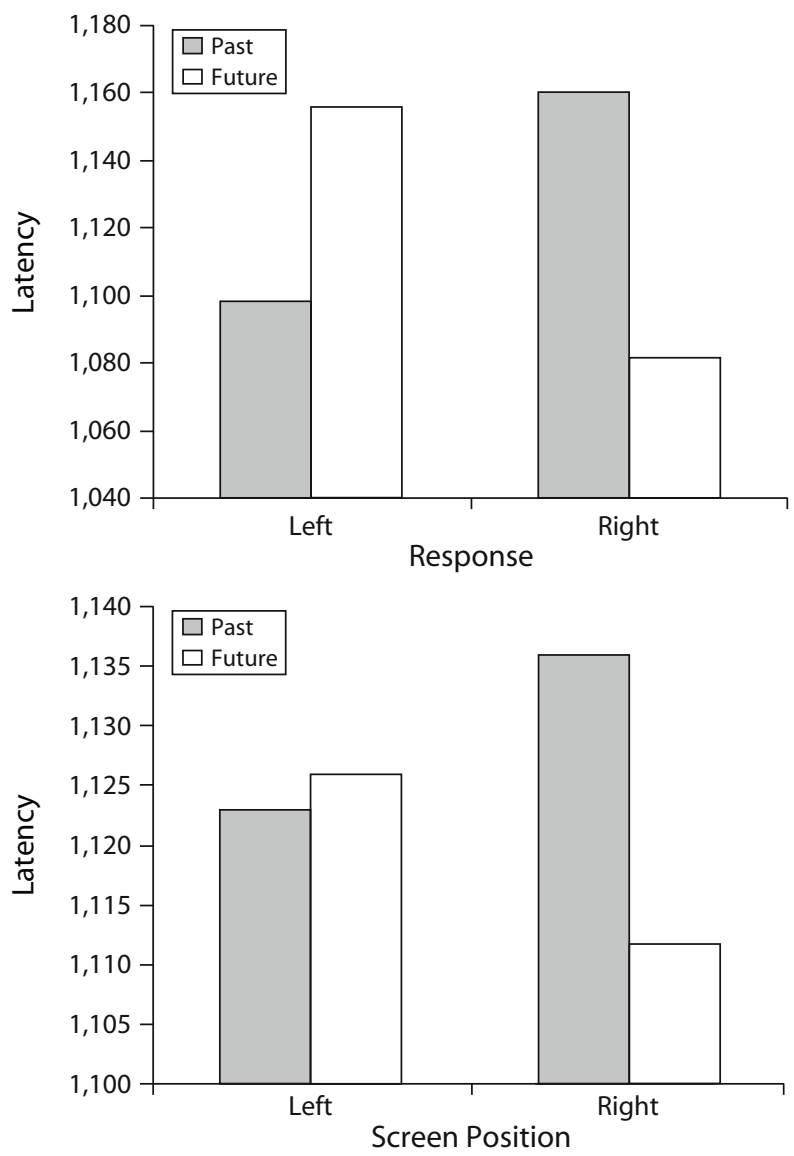

Figure 1. Mean reaction time of correct responses to words with past and future meanings as a function of response position (top panel) and screen position (bottom panel).

perimental block, the words in one list were presented to the left of the fixation point, and words in the other list were presented to the right of fixation. In the second block, lists switched positions. Assignment of list to position was also counterbalanced across participants. Therefore, each participant saw the same word four times, once in each screen position within each key-to-response mapping.

Within each trial, a fixation point was presented centered on the screen during $500 \mathrm{msec}$, followed by a word that remained in view until a response was detected. The fixation point remained in view during the whole duration of the trial. Words were presented centered on a point located $4 \mathrm{~cm}$ to the right or the same distance to the left of the fixation point. After a response was detected, there was a blank intertrial interval of $500 \mathrm{msec}$.

\section{Design and Data Analysis}

Reaction time (RT) on correct trials and accuracy were recorded and analyzed as a function of three factors in a fully crossed factorial design: meaning (past or future) $\times$ position (left or right screen position) $\times$ key (left or right). Latencies below $250 \mathrm{msec}$ and above 2,500 msec were considered outliers and discarded.

\section{RESULTS}

Overall error rate was $10 \%$. Out of the correct trials, $2.87 \%$ were outliers and were also discarded from the RT analyses. Table 1 shows mean results per condition. There was a clear cross-over interaction between meaning and key $\left[F(1,31)=9.14, M S_{\mathrm{e}}=31,928.16, p<.01\right.$; see Fig- ure 1]. There was a trend toward an interaction between meaning and screen position $\left[F(1,31)=2.63, M S_{\mathrm{e}}=\right.$ $4,459.20, p=.11$; see also Figure 1]. No other main effect or interaction was reliable in the analysis of latencies. The direction of both interactions agreed with our hypotheses: Congruent trials (past words presented on the left or responded to with the left key, and future words presented on the right or responded to with the right key) were faster than incongruent trials by $67 \mathrm{msec}$ with respect to the response position dimension, and by $14 \mathrm{msec}$ with respect to the screen position dimension. Both past and future words appeared to be affected by spatial dimensions to a similar extent (see Table 1 and Figure 1).

The analysis of accuracy data showed a clear position $\times$ meaning interaction $\left[F(1,31)=11.56, M S_{\mathrm{e}}=\right.$ $0.0015, p<.01]$. Of less relevance to our hypotheses, past words were also less accurate than were future words $\left[F(1,31)=7.38, M S_{\mathrm{e}}=0.0052, p<.05\right]$. No other $F$ was significant at the .05 level. Although the interaction between meaning and key was not significant, the pattern of means was in the expected direction (responses were $0.5 \%$ more accurate in the congruent mapping; see Table 1), showing that there were no speed-accuracy trade-offs.

Finally, nowhere did the interaction between screen position and key reach or even approach significance, suggesting that there were no Simon-type effects in our data. This result is consistent with the literature that shows Simon effects only in the fast section of the RT distribution, with slower RTs leading to null or even reversed effects (see, e.g., Mapelli, Rusconi, \& Umiltà, 2003).

\section{DISCUSSION}

When participants judged the past or future reference of written words, they were affected by spatial characteristics of the task that were completely irrelevant to their processing goals. The shape of this interaction between space and time took the form that was predicted by the left-right conceptual metaphor hypothesis. Responses were faster when past and future time were mapped onto left and right keys, respectively, than when the opposite mapping was used. This result is a direct analogy in the domain of time of the SNARC effect that was found by Dehaene et al. (1993). Moreover, words were somewhat faster and clearly more accurate when they were presented on the screen side that was congruent with their temporal meaning: past words on the left, and future words on the right.

The fact that both stimulus and response position are irrelevant to the task of judging temporal reference suggests that thinking of the abstract domain of time leads to

Table 1

Mean Latency (RT, in Milliseconds) and Percent Errors (\%E) per Condition

\begin{tabular}{|c|c|c|c|c|c|c|c|c|}
\hline \multirow[b]{4}{*}{ Key } & \multicolumn{8}{|c|}{ Screen Position } \\
\hline & \multicolumn{4}{|c|}{ Left } & \multicolumn{4}{|c|}{ Right } \\
\hline & \multicolumn{2}{|c|}{ Past } & \multicolumn{2}{|c|}{ Future } & \multicolumn{2}{|c|}{ Past } & \multicolumn{2}{|c|}{ Future } \\
\hline & RT & $\% \mathrm{E}$ & RT & $\% \mathrm{E}$ & RT & $\% \mathrm{E}$ & RT & $\% \mathrm{E}$ \\
\hline Left & 1,101 & 11.1 & 1,159 & 10.3 & 1,095 & 12.9 & 1,153 & 9.6 \\
\hline Right & 1,144 & 9.9 & 1,093 & 9.3 & 1,176 & 12 & 1,071 & 6.8 \\
\hline
\end{tabular}


the activation of concrete spatial concepts, as predicted by the conceptual metaphor view (Lakoff \& Johnson, 1980). Moreover, present data support the idea that there exists a specific mapping between past time and left space and future time and right space.

An alternative account raised by a reviewer of this paper (Daniel Casasanto) suggests that participants may have noted that the stimuli constitute ordered pairs (e.g., I spoke-I will speak). Gevers et al. $(2003,2004)$ found that ordinal sequences like months, letters, and days of the week tend to be spatialized from left to right. Thus, it might be the ordinal relation between the members of each pair and not their temporal reference that generates the left-right spatialization in our data. Although it may be impossible to discriminate the correct explanation in the context of the present experiment, some aspects of the data suggest that a purely ordinal representation of experimental word pairs cannot be the whole story. This account predicts that the congruency effect should be stronger in the second half of the experiment, because the repeated experience with the stimulus pairs is supposed to induce and strenghthen their representation as ordered pairs. Contrary to such expectations, follow-up analyses failed to find any sign that the effects of either key or screen position congruency increased toward the second half of the experiment. This result makes the suggested alternative hypothesis less attractive.

Present results are inconclusive with respect to several issues. First, they do not allow us to assert whether the conceptual metaphor being used is an ego-reference-point or a time-reference-point metaphor, in Núñez and Sweetser's (2006) terms. Future research will address this question, as well as explore other aspects of the inferential structure of the metaphor, such as whether there is a continuous relation between space and time along the whole mental "time line" instead of just the dichotomous left-right relation presently reported. The present data do not allow us to assert why the mapping takes this form and not, say, past-right and future-left; however, we suggest (for the reasons discussed in the introduction) that the cause lies in repeated exposure to written language and graphic conventions in left-to-right written languages. A direct test of this hypothesis will have to await future cross-linguistic research. If supported by the evidence, future theorizing on conceptual metaphor acquisition will need to consider one more route to the establishment of cross-domain mappings. So far, exposure to habitual language has been considered the main determinant of the mappings attested in a given culture (Boroditsky, 2001; Casasanto et al., 2004; Slobin, 1996). Exposure to written language and graphic conventions should be considered another important determinant.

Present results not only agree with expectations from the conceptual metaphor view, but also broaden the scope of this research program beyond its original boundaries. ${ }^{1}$ The entire cognitive linguistics enterprise is concerned with inferring patterns of thought from the analysis of patterns of language use. By showing that time can be spatialized in ways that have no corresponding reflection in language, our work extends the relevant cognitive linguistics database from linguistic conventions to the whole spectrum of cultural conventions.
When the present study is considered in the context of the literature on conceptual metaphoric representation, a primary question that is left unanswered concerns the existence of alternative and often incompatible mappings for the same conceptual domains in the same minds. How can a back-front and a left-right mapping of time onto space coexist in the minds of Westerners? What are the factors that lead to the activation of one mapping or another? Do conflicts arise and how are they resolved? These questions are thought by many scholars to be key considerations for the conceptual metaphor view (Rakova, 2002). Some studies are already approaching this issue by examining the conditions that lead to the activation of alternative mappings (Boroditsky, 2000; Boroditsky \& Ramscar, 2002). In a replication and extension of the present results, Torralbo, Santiago, \& Lupiáñez (2006) have shown that attention plays an important role in selecting the relevant space-time mapping. They manipulated the attention that was payed to either the left-right or back-front spatial frames of reference through automatic means (changing task requirements) and found that the attended frame is used to guide the mapping of space onto time. Attention to the back-front frame leads to the past being projected onto back space and the future being projected onto front space, and blocks the projection of past and future onto left and right space. The converse occurs when the left-right frame is focused. Recently, Santiago, Román, and Ouellet (2007) obtained similar results when attention was manipulated through voluntary-endogenous means (task instructions). Thus, attentional mechanisms may lie at the heart of the solution to this question. Torralbo et al. (2006; see also Santiago et al., 2007) outline a theoretical framework that is able to account for prior results, draw a number of novel predictions, and provide supportive evidence.

\section{AUTHOR NOTE}

The authors are indebted to Antonio Román, Nieves Rodríguez, and Ouafa Bouachra for running the experiment and being such enthusiastic students. We would like to thank Arthur Glenberg and Daniel Casasanto for their comments on a prior draft of this article. Address correspondence to J. Santiago, Dept. de Psicología, Universidad de Granada, Campus de Cartuja, Granada, Spain (e-mail: santiago@ugr.es).

\section{REFERENCES}

Alverson, H. (1994). Semantics and experience: Universal metaphors of time in English, Mandarin, Hindi, and Sesotho. Baltimore: Johns Hopkins University Press.

Boroditsky, L. (2000). Metaphoric structuring: Understanding time through spatial metaphors. Cognition, 75, 1-28.

BorodiTSKY, L. (2001). Does language shape thought? Mandarin and English speakers' conceptions of time. Cognitive Psychology, 43, 1-22.

Boroditsky, L., \& RAMSCAR, M. (2002). The roles of body and mind in abstract thought. Psychological Science, 13, 185-189.

Casasanto, D., Boroditsky, L., Phillips, W., Greene, J., Goswami, S., Bocanegra-Thiel, S., ET AL. (2004). How deep are effects of language on thought? Time estimation in speakers of English, Indonesian, Greek, and Spanish. In K. Forbus, D. Gentner, \& T. Regier (Eds.), Proceedings of the 26th Annual Meeting of the Cognitive Science Society (pp. 186-191). Mahwah, NJ: Erlbaum.

Chatterjee, A., Maher, L. M., Gonzalez-Rothi, L., \& Heilman, K. M. (1995). Asyntactic thematic role assignment: The use of a temporal-spatial strategy. Brain \& Language, 49, 125-139.

Chatterjee, A., Southwood, M. H., \& Basilico, D. (1999). Verbs, events and spatial representations. Neuropsychologia, 37, 395-402. 
Clark, H. H. (1973). Space, time, semantics and the child. In T. E. Moore (Ed.), Cognitive development and the acquisition of language. (pp. 27-63). New York: Academic Press.

Dehaene, S., Bossini, S., \& Giraux, P. (1993). The mental representation of parity and number magnitude. Journal of Experimental Psychology: General, 122, 371-396.

Emmorey, K. (2001). Space on hand: The exploitation of signing space to illustrate abstract thought. In M. Gattis (Ed.), Spatial schemas and abstract thought (pp. 147-174). Cambridge, MA: MIT Press.

Gevers, W., Reynvoet, B., \& Fias, W. (2003). The mental representation of ordinal sequences is spatially organized. Cognition, 87, B87-B95.

Gevers, W., Reynvoet, B., \& Fias, W. (2004). The mental representation of ordinal sequences is spatially organized: Evidence from days of the week. Cortex, 40, 171-172.

JoHnson, M. (1987). The body in the mind. Chicago: University of Chicago Press.

KLeIN, H. (1987). Time in Toba. Word, 38, 173-185.

Lakoff, G., \& Johnson, M. (1980). Metaphors we live by. Chicago: University of Chicago Press.

Lakoff, G., \& Johnson, M. (1999). Philosophy in the flesh: The embodied mind and its challenge to Western thought. New York: Basic Books.

Levine, R. (1997). A geography of time. New York: Basic Books.

MAAs, A., \& Russo, A. (2003). Directional bias in the mental representation of spatial events: Nature or culture? Psychological Science, 14, 296-301.

Maher, L. M., Chatterjee, A., Gonzalez-Rothi, L., \& Heilman, K. M. (1995). Agrammatic sentence production: The use of a temporal-spatial strategy. Brain \& Language, 49, 105-124.

Malotki, E. (1983). Hopi time: A linguistic analysis of temporal concepts in the Hopi language. Berlin: Mouton.

Mandler, J. M. (1992). How to build a baby: II. Conceptual primitives. Psychological Review, 99, 587-604.

Mapelli, D., Rusconi, E., \& Umiltà, C. (2003). The SNARC effect: An instance of the Simon effect? Cognition, 88, B1-B10.

McGlone, M. S., \& Harding, J. L. (1998). Back (or forward?) to the future: The role of perspective in temporal language comprehension. Journal of Experimental Psychology: Learning, Memory, \& Cognition, 24, 1211-1223.
Núñez, R. E., \& SweEtser, E. (2006). With the future behind them: Convergent evidence from Aymara language and gesture in the crosslinguistic comparison of spatial construals of time. Cognitive Science, 30, 401-450.

ÖZÇALISKAN, S. (2003). Metaphorical motion in crosslinguistic perspective: A comparison of English and Turkish. Metaphor \& Symbol, 18, 189-228.

RADDEN, G. (2004). The metaphor TIME AS SPACE across languages. In N. Baumgarten et al. (Eds.), Übersetzen, interkulturelle Kommunikation, Spracherwerb und Sprachvermittlung —Das Leben mit Mehreren Sprachen: Festschrift für Juliane House zum 60. Geburtstag (pp. 225238). Bochum: Aks-verlag.

RAKova, M. (2002). The philosophy of embodied realism: A high price to pay? Cognitive Linguistics, 13, 215-244.

Santiago, J., Román, A., \& Oullet, M. (2007). Conceptual metaphors and cognitive flexibility: A review and a theory. Manuscript in preparation.

SCHNEIDER, W. (1988). Micro Experimental Laboratory: An integrated system for IBM PC compatibles. Behavior Research Methods, Instruments, \& Computers, 20, 206-217.

SLOBIN, D. (1996). From "thought and language" to "thinking for speaking.” In J. J. Gumperz, \& S. C. Levinson (Eds.), Rethinking linguistic relativity (pp. 70-96). Cambridge: Cambridge University Press.

Torralbo, A., Santiago, J., \& Lupiáñez, J. (2006). Flexible conceptual projection of time onto spatial frames of reference. Cognitive Science, 30, 745-757.

Tversky, B., Kugelmass, S., \& Winter, A. (1991). Cross-cultural and developmental trends in graphic productions. Cognitive Psychology, 23, 515-557.

Yu, N. (1998). The contemporary theory of metaphor: A perspective from Chinese. Amsterdam: Benjamins.

ZEBIAN, S. (2005). Linkages between number concepts, spatial thinking, and directionality of writing: The SNARC effect and the reverse SNARC effect in English and Arabic monoliterates, biliterates, and illiterate Arabic speakers. Journal of Cognition \& Culture, 5, 165-190.

\section{NOTE}

1. We thank Daniel Casasanto for bringing this point up.

APPENDIX

Materials (Translations in Brackets)

\begin{tabular}{ll}
\hline \multicolumn{1}{c}{ Past } & \multicolumn{1}{c}{ Future } \\
\hline pasado (past) & futuro (future) \\
después (after) \\
recientemente (recently) & próximamente (nearly) \\
anteriormente (previously) & posteriormente (subsequently) \\
ayer (yesterday) & mañana (tomorrow) \\
anteayer (before yesterday) & enseguida (next) \\
antiguamente (formerly) & inmediatamente (immediately) \\
dijo (he said) & dirá (he will say) \\
tuvimos (we had) & tendremos (we will have) \\
hablé (I spoke) & hablaré (I will speak) \\
pudimos (we could) & podremos (we will be able to) \\
buscásteis (you-plural looked for) & buscaremos (we will look for) \\
probásteis (you-plural tried) & probaréis (you-plural will try) \\
fuiste (you-singular went) & irás (you-singular will go) \\
aparecí (I showed up) & apareceré (I will show up) \\
vió (he saw) & verá (he will see) \\
pensé (I thought) & pensaré (I will think) \\
miré (I looked) & miraré (I will look) \\
preguntó (he asked) & preguntará (he will ask) \\
decidisteis (you-plural decided) & decidiréis (you-plural will decide) \\
quisimos (we wanted) & querremos (we will want) \\
hiciste (you-singular made) & harás (you-singular will make) \\
creiste (you-singular believed) & creerás (you-singular will believe) \\
condujimos (we drove) & conduciremos (we will drive) \\
\hline &
\end{tabular}

(Manuscript received March 30, 2006;

revision accepted for publication July 10, 2006.) 